\title{
HIGH ENERGY TRANSIENT EXPLORER 2 OBSERVATIONS OF THE EXTREMELY SOFT X-RAY FLASH XRF 020903
}

T. Sakamoto, ${ }^{1,2,3}$ D. Q. Lamb ${ }^{4}$ C. Graziani, ${ }^{4}$ T. Q. Donaghy,${ }^{4}$ M. Suzuki,${ }^{1}$ G. Ricker, ${ }^{5}$ J.-L. Atteia, ${ }^{6}$ N. Kawai, ${ }^{1,2}$ A. Yoshida, ${ }^{2,7}$ Y. Shirasaki, ${ }^{8}$ T. Tamagawa, ${ }^{2}$ K. Torit, ${ }^{2}$ M. Matsuoka, ${ }^{9}$ E. E. Fenimore, ${ }^{3}$ M. Galassi,${ }^{3}$ T. Tavenner, ${ }^{10}$ J. Doty,${ }^{5}$ R. Vanderspek, ${ }^{5}$ G. B. Crew, ${ }^{5}$ J. Villasenor, ${ }^{5}$ N. Butler, ${ }^{5}$ G. Prigozhin, ${ }^{5}$ J. G. Jernigan, ${ }^{11}$ C. Barraud, ${ }^{6}$ M. Boer, ${ }^{12}$ J.-P. Dezalay, ${ }^{12}$ J.-F. Olive, ${ }^{12}$ K. Hurley, ${ }^{11}$ A. Levine, ${ }^{5}$ G. Monnelly, ${ }^{5}$ F. Martel ${ }^{5}$ E. Morgan, ${ }^{5}$ S. E. Woosley, ${ }^{13}$ T. Cline, ${ }^{14}$ J. Braga, ${ }^{15}$ R. Manchanda, ${ }^{16}$ G. Pizzichini, ${ }^{17}$ K. Takagishi, ${ }^{18}$ and M. Yamauchi ${ }^{18}$ Received 2003 April 24; accepted 2003 November 7

\begin{abstract}
We report High Energy Transient Explorer 2 (HETE-2) Wide Field X-Ray Monitor/French Gamma Telescope observations of the X-ray flash XRF 020903. This event was extremely soft: the ratio $\log \left(S_{\mathrm{X}} / S_{\gamma}\right)=0.7$, where $S_{\mathrm{X}}$ and $S_{\gamma}$ are the fluences in the 2-30 and 30-400 keV energy bands, is the most extreme value observed so far by HETE-2. In addition, the spectrum has an observed peak energy of $E_{\text {peak }}^{\text {obs }}<5.0 \mathrm{keV}(99.7 \%$ probability upper limit), and no photons were detected above $\sim 10 \mathrm{keV}$. The burst is shorter at higher energies, which is similar to the behavior of long gamma-ray bursts (GRBs). We consider the possibility that the burst lies at very high redshift and that the low value of $E_{\text {peak }}^{\text {obs }}$ is due to the cosmological redshift, and show that this is very unlikely. We find that the properties of XRF 020903 are consistent with the relation between the fluences $S(7-30 \mathrm{keV})$ and $S(30-400 \mathrm{keV})$, found by Barraud et al. for GRBs and X-ray-rich GRBs, and are consistent with the extension by a decade of the hardness-intensity correlation found by the same authors. Assuming that XRF 020903 lies at a redshift $z=0.25$, as implied by the host galaxy of the candidate optical and radio afterglows of this burst, we find that the properties of XRF 020903 are consistent with an extension by a factor $\sim 300$ of the relation between the isotropic-equivalent energy $E_{\text {iso }}$ and the peak $E_{\text {peak }}$ of the $\nu F_{\nu}$ spectrum (in the source frame of the burst) found by Amati et al. for GRBs. The results presented in this paper therefore provide evidence that X-ray flashes (XRFs), X-ray-rich GRBs, and GRBs form a continuum and are a single phenomenon. The results also impose strong constraints on models of XRFs and X-ray-rich GRBs.
\end{abstract}

Subject headings: gamma rays: bursts - X-rays: bursts — X-rays: individual (GRB 020903)

On-line material: color figures

${ }^{1}$ Department of Physics, Tokyo Institute of Technology, 2-12-1 Ookayama, Meguro-ku, Tokyo 152-8551, Japan.

2 RIKEN (Institute of Physical and Chemical Research), 2-1 Hirosawa, Wako, Saitama 351-0198, Japan.

${ }^{3}$ Los Alamos National Laboratory, P.O. Box 1663, Los Alamos, NM 87545 .

${ }^{4}$ Department of Astronomy and Astrophysics, University of Chicago, Chicago, IL 60637.

${ }^{5}$ Center for Space Research, Massachusetts Institute of Technology, 70 Vassar Street, Cambridge, MA 02139.

${ }^{6}$ Laboratoire d'Astrophysique, Observatoire Midi-Pyreńeés, 14 Avenue E. Belin, 31400 Toulouse, France.

${ }^{7}$ Department of Physics, Aoyama Gakuin University, Chitosedai 6-16-1, Setagaya-ku, Tokyo 157-8572, Japan.

${ }^{8}$ National Astronomical Observatory, Osawa 2-21-1, Mitaka, Tokyo 181-8588, Japan.

${ }^{9}$ Tsukuba Space Center, National Space Development Agency of Japan, Tsukuba, Ibaraki 305-8505, Japan.

${ }^{10}$ Department of Astronomy, New Mexico State University, 1320 Frenger Mall, Las Cruces, NM 88003-8001.

${ }^{11}$ University of California at Berkeley, Space Sciences Laboratory, Berkeley, CA 94720-7450.

${ }^{12}$ Centre d'Etude Spatiale des Rayonnements, CNRS/UPS, B.P.4346, 31028 Toulouse Cedex 4, France.

${ }^{13}$ Department of Astronomy and Astrophysics, University of California at Santa Cruz, 477 Clark Kerr Hall, Santa Cruz, CA 95064.

${ }^{14}$ NASA Goddard Space Flight Center, Greenbelt, MD 20771.

15 Instituto Nacional de Pesquisas Espaciais, Avenida Dos Astronautas 1758, Saõ Josédos Campos 12227-010, Brazil.

16 Department of Astronomy and Astrophysics, Tata Institute of Fundamental Research, Homi Bhabha Road, Bombay 400 005, India.

${ }_{17}^{17}$ IASF/CNR Sezione di Bologna, via Gobetti 101, 40129 Bologna, Italy.

${ }^{18}$ Faculty of engineering, Miyazaki University, Gakuen Kibanadai Nishi, Miyazaki 889-2192, Japan.

\section{INTRODUCTION}

Gamma-ray bursts (GRBs) that have a large fluence in the $\mathrm{X}$-ray energy band $(2-30 \mathrm{keV})$ relative to the gamma-ray energy band (30-400 keV) are receiving increased attention. The Burst and Transient Source Experiment (BATSE) on board the Compton Gamma Ray Observatory detected 2704 GRBs (Paciesas et al. 1999). The spectra of 156 bright bursts exhibit a distribution of low-energy power-law indices, $\alpha$, whose centroid is $\sim-1$, and a distribution of observed break energies, $E_{\text {break }}^{\text {obs }}$, whose centroid is $\approx 230 \mathrm{keV}$ (Preece et al. $2000)$, where $E_{\text {break }}^{\text {obs }}=(\alpha-\beta)(2+\alpha)^{-1} E_{\text {peak }}^{\text {obs }}$. Here $\alpha, \beta$, and $E_{\text {peak }}^{\text {obs }}$ are the slope of the low-energy power-law index, the high-energy power-law index, and the energy of the peak of the $\nu F_{\nu}$ spectrum of the Band function (Band et al. 1993), an expression that satisfactorily represents the spectra of almost all GRBs. In contrast, $36 \%$ of the bright bursts observed by Ginga have peak energies $E_{\text {peak }}^{\text {obs }}$ in their photon number spectrum at a few $\mathrm{keV}$ and large X-ray to $\gamma$-ray fluence ratios (Strohmayer et al. 1998).

The BeppoSAX Wide Field Camera (WFC) detected events that are very similar to the soft Ginga GRBs; these events have been termed "X-ray flashes" (XRFs) (Heise et al. 2000). ${ }^{19}$ The energy flux of these XRFs lies in the range $10^{-8}$

\footnotetext{
${ }^{19}$ Throughout this paper, we define "X-ray-rich" GRBs and XRFs as those events for which $\log \left[S_{\mathrm{X}}(2-30 \mathrm{kev}) / S_{\gamma}(30-400 \mathrm{kev})\right]>-0.5$ and 0.0 , respectively.
} 
to $10^{-7} \mathrm{ergs}^{-2} \mathrm{~s}^{-1}$, and the low-energy photon index $\alpha$ of their spectra ranges from -3 to -1.2 . The sky distribution of $\mathrm{XRFs}$ is consistent with isotropy, and there is no evidence that the sources are Galactic. The XRFs have $t_{90}$ durations between 10 and $200 \mathrm{~s}$. The event rate of XRFs detected by the WFC is 3.4 events $\mathrm{yr}^{-1}$. Clarifying the connection between XRFs and GRBs could provide a breakthrough in our understanding of the prompt emission of GRBs.

Kippen et al. (2002) made a detailed spectral comparison of GRBs and XRFs, using a sample of 18 GRBs that were observed by BATSE and a sample of nine XRFs that were observed by both the WFC and BATSE. According to their joint analysis of WFC/BATSE spectral data, the low-energy and high-energy photon indices of XRFs are -1 and $\sim-2.5$, respectively, which are no different from those of GRBs. On the other hand, XRFs have much lower values of $E_{\text {peak }}^{\text {obs }}$ than do GRBs. Thus the only temporal or spectral difference between GRBs and XRFs appears to be that XRFs have lower $E_{\text {peak }}^{\text {obs }}$ values. Kippen et al. therefore suggest that XRFs might represent an extension of the GRB population to events with low peak energies. Analyzing 35 High Energy Transient 2 (HETE-2) GRBs seen by French Gamma Telescope (FREGATE), Barraud et al. (2003) demonstrate that the spectral properties of "X-ray-rich" GRBs form a continuum with those of ordinary GRBs and suggest that XRFs may represent a further extension of this continuum.

BATSE's low-energy threshold of $\sim 20 \mathrm{keV}$ made it difficult for BATSE to detect XRFs. Ginga and BeppoSAX had the capability of detecting XRFs; however, Ginga could not determine the direction of the burst and the BeppoSAX GRBM had difficulty in triggering on XRFs. Consequently, these missions could not carry out in depth investigations of XRFs. In contrast, HETE-2 (Ricker et al. 2003) has the ability to trigger on and localize XRFs and to study their spectral properties, using the Wide-Field X-Ray Monitor (WXM; $2-$ $25 \mathrm{keV}$ energy band; Kawai et al. 2003) and the FREGATE (6-400 keV energy band; Atteia et al. 2003), which have energy thresholds of a few $\mathrm{keV}$.

In this paper we report the detection and localization of XRF 020903 by HETE-2 (Ricker et al. 2002) and present the results of a detailed study of its properties. Since this event was extremely soft and there was very little signal (a $\sim 2 \sigma$ excess in the best selected energy range) in FREGATE, we focus our analysis on the WXM temporal and spectral data for the event.

\section{OBSERVATIONS}

\subsection{Localization}

XRF 020903 was detected with the HETE-2 WXM and the Soft X-ray Camera (SXC; $0.5-10 \mathrm{keV}$ energy band; Villasenor et al. 2003) instruments at 10:05:37.96 UT on 2002 September 3 (Ricker et al. 2002). The WXM flight localization was correct, but it was not sent out because HETE-2 was pointing at the Galactic bulge region at the time and WXM triggers were therefore not being sent to the GCN in order not to overwhelm the astronomical community with X-ray burst localizations. A GCN Notice reporting the localization of the burst, based on ground analysis (Graziani et al. 2003; Shirasaki et al. 2003) of the WXM data, was sent out 231 minutes after the burst.

The WXM localization can be expressed as a $90 \%$ confidence circle that is 16.6 in radius and is centered at R.A. = $22^{\mathrm{h}} 49^{\mathrm{m}} 25^{\mathrm{s}}$, decl. $=-20^{\circ} 53^{\prime} 59^{\prime \prime}(\mathrm{J} 2000.0)$. A localization of the burst based on ground analysis (Monnelly et al.
2003) of the SXC data was distributed as a GCN Notice about $7 \mathrm{hr}$ after the burst. Only a one-dimensional localization was possible using the SXC data, but this significantly reduced the area of the localization region for XRF 020903. The improved localization produced by combining the SXC and WXM localizations can be described as a $90 \%$ confidence quadrilateral that is $4^{\prime}$ in width and $\sim 31^{\prime}$ in length (see Fig. 1). It is centered at R.A. $=22^{\mathrm{h}} 49^{\mathrm{m}} 01^{\mathrm{s}}$, decl. $=-20^{\circ} 55^{\prime} 47^{\prime \prime}(\mathrm{J} 2000.0)$, and its four corners lie at (R.A., decl.) $=\left(22^{\mathrm{h}} 48^{\mathrm{m}} 4800\right.$, $\left.-20^{\circ} 39^{\prime} 360\right), \quad\left(22^{\mathrm{h}} 48^{\mathrm{m}} 3360, \quad-20^{\circ} 42^{\prime} 360\right), \quad\left(22^{\mathrm{h}} 49^{\mathrm{m}} 1080\right.$, $\left.-21^{\circ} 10^{\prime} 120\right)$, and $\left(22^{\mathrm{h}} 49^{\mathrm{m}} 3000,-21^{\circ} 10^{\prime} 480\right)(\mathrm{J} 2000.0)$.

Detections of candidate optical and radio afterglows of XRF 020903, and the host galaxy of the candidate optical and radio afterglows, have been reported. Soderberg et al. (2002) discovered an optical transient within the HETE-2 SXC+WXM localization region at R.A. $=22^{\mathrm{h}} 48^{\mathrm{m}} 4234$, decl. $=-20^{\circ} 46^{\prime} 09.3^{\prime \prime}$, using the Palomar $5 \mathrm{~m}$ telescope. These authors mention that the optical transient brightened by $\sim 0.3-$ 0.4 mag between about 7 and 24 days after the XRF and suggest that the rebrightening might be due to an associated supernova. However, the optical transient apparently faded by over a magnitude only 3 days later (Covino et al. 2002). Spectroscopic observations of the optical transient, using the Magellan $6.5 \mathrm{~m}$ Baade and Clay telescopes, detected narrow emission lines from an underlying galaxy at a redshift $z=0.25 \pm 0.01$, suggesting that the host galaxy of the optical transient is a star-forming galaxy (Soderberg et al. 2002; see also Chornock \& Filippenko 2002). A fading bright radio source at the position of optical transient was detected using the Very Large Array (Berger et al. 2002). Hubble Space Telescope observations of the XRF 020903 field reveal the optical transient and show that its host galaxy is an irregular galaxy, possibly with four interacting components (Levan

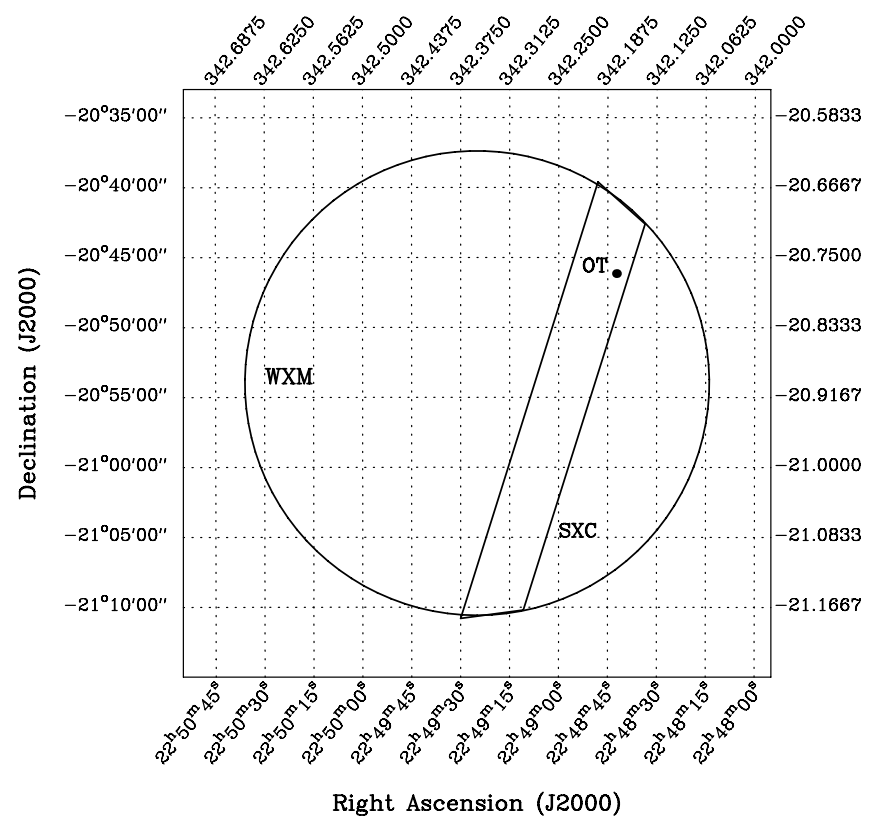

FIG. 1.-HETE-2 WXM/SXC localization for XRF 020903. The circle is the $90 \%$ confidence region for the WXM localization and the beltlike region is the portion of the $90 \%$ confidence region for the one-dimensional SXC localization that lies within the WXM 90\% confidence circle. The final localization is the intersection of the WXM and SXC localizations (Ricker et al. 2002). The point labeled "OT" is the location of the candidate optical (Soderberg et al. 2002) and radio (Berger et al. 2002) afterglows of XRF 020903. [See the electronic edition of the Journal for a color version of this figure.] 
et al. 2002). These detections likely represent the first discoveries of the optical and radio afterglows, together with the host galaxy, of an XRF.

In our analysis of the prompt emission of XRF 020903, we apply a "cut" to the WXM photon time- and energy-tagged data (TAG data), using only the photons from the pixels on the three wires in the X-detectors (XA0, XA1, and XA2) and the four wires in the Y-detectors (YA1, YA2; YB0, YB1) that were illuminated by the burst and that maximize the signalto-noise ratio $(\mathrm{S} / \mathrm{N})$ of the burst light curve, in the same manner as we did for GRB 020531 (Lamb, Donaghy, \& Graziani 2003a). We use this optimized TAG data when performing our temporal and spectral analyses of this event.

\subsection{Temporal Properties}

Figure 2 shows the light curve of XRF 020903 in four WXM energy bands. The time history of the burst in the $2-5$ and $5-10 \mathrm{keV}$ energy bands has two peaks. Clearly, there is no significant flux above $10 \mathrm{keV}$. Table 1 gives the $t_{50}$ and $t_{90}$ durations of the burst in the 2-5,5-10, and 2-10 keV energy bands. The duration of the burst is longer in the lower energy band; this trend is similar to that seen in long bright GRBs (Fenimore et al. 1995).

\subsection{Spectrum}

As we have seen, the light curve of XRF 020903 shows two peaks, the first occurring in the time interval $0-8 \mathrm{~s}$ and the second occurring in the time interval $8-13 \mathrm{~s}$. The $\mathrm{S} / \mathrm{N}$ of the first peak is much higher than that of the second. In addition, inspection of the burst light curve in the $2-5$ and $5-10 \mathrm{keV}$ energy bands suggests that the second peak is much softer than the first. For these reasons, we analyze the spectrum of the burst in three time intervals: $0-8 \mathrm{~s}, 8-13 \mathrm{~s}$, and the total duration of the burst, $0-13 \mathrm{~s}$. The background region that we use is $40 \mathrm{~s}$ in duration and starts $45 \mathrm{~s}$ before the burst.

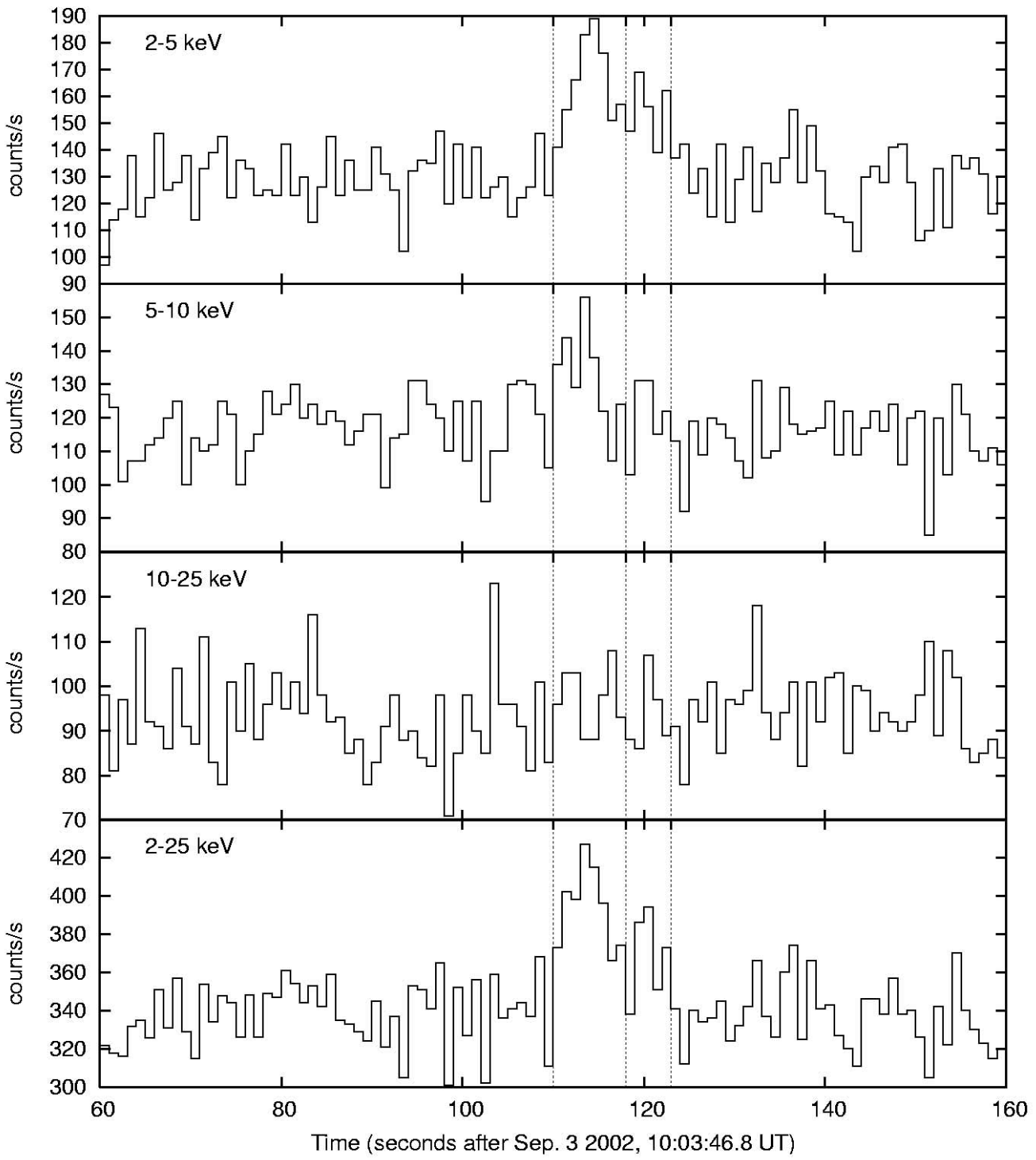

FIG. 2.-Light curve of XRF 020903 in four WXM energy bands: $2-5,5-10,10-25$, and 2-25 keV (top to bottom). The light curve is binned in one second bins. The vertical dotted lines show the $0-8$ and $8-13$ s time intervals bracketing the first and second peaks of the burst light curve. We have performed model fits to the spectra of the burst during these two time intervals and to the entire duration of the burst $(0-13 \mathrm{~s})$. 
TABLE 1

Temporal Properties of XRF 020903

\begin{tabular}{|c|c|c|}
\hline $\begin{array}{c}\text { Energy Band } \\
(\mathrm{keV})\end{array}$ & $\begin{array}{l}t_{50} \\
(\mathrm{~s})\end{array}$ & $\begin{array}{l}t_{90} \\
(\mathrm{~s})\end{array}$ \\
\hline $2-5$ & $5.8 \pm 0.9$ & $10.6 \pm 0.2$ \\
\hline $5-10 \ldots \ldots \ldots \ldots \ldots \ldots \ldots \ldots$ & $2.4 \pm 0.2$ & $4.3 \pm 2.2$ \\
\hline $2-10 \ldots \ldots \ldots \ldots \ldots \ldots \ldots \ldots$ & $4.9 \pm 0.6$ & $9.8 \pm 0.6$ \\
\hline
\end{tabular}

Note.-The quoted errors correspond to $\pm 1 \sigma$.

The WXM detector response matrix has been well-calibrated using observations of the Crab Nebula (Shirasaki et al. 2002). In the spectral fits, we include only the photons that registered on the four wires in the X-detectors and the five wires in the Y-detectors that were illuminated by the burst, as mentioned above. Since the variation in the gain is not uniform at the ends of the wires in the WXM detectors (Shirasaki et al. 2000), we use only the photon counts that registered in the central $\pm 50 \mathrm{~mm}$ region of the wires to construct the spectra of the burst. We include all of the photons that register in the central regions of these wires (i.e., we use the full $2-25 \mathrm{keV}$ energy range of the WXM). The relation between pulse height and energy in the WXM is nonlinear and is different for each wire. In order to extract the strongest possible constraints on the parameters of the spectral models we consider, we treat each individual WXM wire separately but take the normalizations on all wires to be the same. For the same reason, we do not rebin any of the pulse height channels in the WXM and in the FREGATE, and we carry out a set of fits for the total duration of the burst (0-13 s) that include the spectral data from both the WXM and the FREGATE. We use the XSPEC version 11.2.0 software package to do the spectral fits.

Table 2 presents the results of our time-resolved and timeintegrated spectral analysis of the burst. In this analysis, we consider the following models: (1) blackbody, (2) power-law model, (3) power-law times exponential (the COMP model in Preece et al. 2000), and (4) Band function (Band et al. 1993). The Galactic value of $N_{\mathrm{H}}$ in the direction of the burst is
$2.3 \times 10^{20} \mathrm{~cm}^{-2}$ (Dickey \& Lockman 1990), which is negligible (i.e., it is undetectable in the WXM energy range). Furthermore, the WXM and FREGATE data do not request $N_{\mathrm{H}}$ as a free parameter (i.e., introducing $N_{\mathrm{H}}$ as an additional free parameter produces only a small change in $\chi^{2}$ ). There is therefore no need to include $N_{\mathrm{H}}$ as a parameter in the spectral fits, and we do not do so. In the Band model fits, we have fixed $\alpha=-1$, which is a typical value for GRBs, in order to better constrain the remaining parameters. All of the models provide acceptable fits to the data; i.e., the data do not request models more complicated than a blackbody or a power law.

However, essentially all GRB spectra are well described by the Band function (Band et al. 1993; Preece et al. 2000), and the analysis of Kippen et al. (2002) shows that at least some XRF spectra are also well described by the Band function. Fits to the WXM data for all three time intervals using the powerlaw model give spectral slopes $\alpha<-2$ with high significance. For example, comparing the minimum value $\chi_{\min }^{2}=53.4$ corresponding to the best-fit value of the spectral slope $\alpha=$ -2.8 and the value $\chi^{2}=60.6$ at $\alpha=-2$ for the power-law fit to the average spectrum of the burst (i.e., the time interval $0-13 \mathrm{~s}$ ), we find that $\Delta \chi^{2}=7.2$ for one additional parameter. Thus, using the maximum likelihood ratio test, $\alpha<-2$ at the $99.3 \%$ confidence level. From this evidence, we conclude that the peak $E_{\text {peak }}$ in $\nu F_{\nu}$ lies near or below $2 \mathrm{keV}$, the lower limit of the energy range of the WXM detectors.

There is evidence of spectral softening between the first and second time intervals. In particular, a power-law fit to the first time interval gives $\alpha_{1}=-2.4_{-0.6}^{+0.5}$ and $\chi_{\min , 1}^{2}=75.1$, while a power-law fit to the second time interval gives $\alpha_{2}=-4.2_{-3.7}^{+1.1}$ and $\chi_{\min , 2}^{2}=71.1$. In contrast, a power-law fit to the first and second time intervals, but with $\alpha=\alpha_{1}=\alpha_{2}$ gives $\alpha=$ $-2.86_{-0.82}^{+0.44}$ and $\chi_{\min }^{2}=152.2$. The first (more complicated) model includes the second model as a special case (i.e., the models are nested). Comparing $\chi_{\min }^{2}$ for the two models, we find that $\Delta \chi^{2}=6.0$ for one additional parameter. Thus, using the maximum likelihood ratio test, there is evidence of spectral softening at the $98.6 \%$ confidence level.

Figure 3 shows a comparison of the observed count spectrum and the count spectrum predicted by the best-fit power-

TABLE 2

Results of Fits to the Spectrum of XRF 020903

\begin{tabular}{|c|c|c|c|c|c|c|}
\hline $\begin{array}{l}\text { Time Region } \\
\text { (s) }\end{array}$ & Model & $\begin{array}{c}k T \\
(\mathrm{keV})\end{array}$ & $\alpha$ & $\beta$ & $\begin{array}{l}E_{\text {peak }}^{\text {obs }} \\
(\mathrm{keV})\end{array}$ & $\begin{array}{c}\chi_{\nu}^{2} \\
\text { (dof) }\end{array}$ \\
\hline \multirow[t]{4}{*}{$0.0-8.0 \ldots \ldots \ldots \ldots \ldots \ldots$} & Blackbody & $1.04_{-0.20}^{+0.24}$ & & & & $1.08(62)$ \\
\hline & Power law & & $-2.4_{-0.6}^{+0.5}$ & & & $1.21(62)$ \\
\hline & Cutoff power law & & -1.0 (fixed) & & $3.1_{-1.1}^{+1.9}$ & $1.14(62)$ \\
\hline & Band & & -1.0 (fixed) & $<-2.4$ & $3.4_{-1.0}^{+1.7}$ & $1.16(61)$ \\
\hline \multirow[t]{4}{*}{$8.0-13.0 \ldots \ldots \ldots \ldots \ldots \ldots .}$. & Blackbody & $0.54_{-0.23}^{+0.23}$ & & & & $1.13(62)$ \\
\hline & Power law & & $-4.2_{-3.7}^{+1.1}$ & & & $1.15(62)$ \\
\hline & Cutoff power law & & -1.0 (fixed) & & $<2.0$ & $1.14(62)$ \\
\hline & Band & & -1.0 (fixed) & $<-3.3$ & $<2.0$ & $1.15(61)$ \\
\hline \multirow[t]{4}{*}{$0.0-13.0 \ldots \ldots \ldots \ldots \ldots \ldots$} & Blackbody & $0.87_{-0.16}^{+0.20}$ & & & & $0.79(62)$ \\
\hline & Power law & & $-2.8_{-0.6}^{+0.5}$ & & & $0.86(62)$ \\
\hline & Cutoff power law & & -1.0 (fixed) & & $2.4_{-0.7}^{+1.2}$ & $0.81(62)$ \\
\hline & Band & & -1.0 (fixed) & $<-2.7$ & $2.4_{-0.7}^{+1.2}$ & $0.82(61)$ \\
\hline $0.0-13.0 \ldots \ldots \ldots \ldots \ldots \ldots$ & Blackbody & $0.90_{-0.17}^{+0.21}$ & & & & 0.85 (177) \\
\hline \multirow[t]{3}{*}{ (with FREGATE) ..... } & Power law & & $-2.6_{-0.5}^{+0.4}$ & & & $0.86(177)$ \\
\hline & Cutoff power law & & -1.0 (fixed) & & $2.6_{-0.8}^{+1.4}$ & 0.85 (177) \\
\hline & Band & & -1.0 (fixed) & $<-2.3$ & $<4.1$ & $0.86(176)$ \\
\hline
\end{tabular}

Note.-The quoted errors correspond to the $90 \%$ confidence region. 

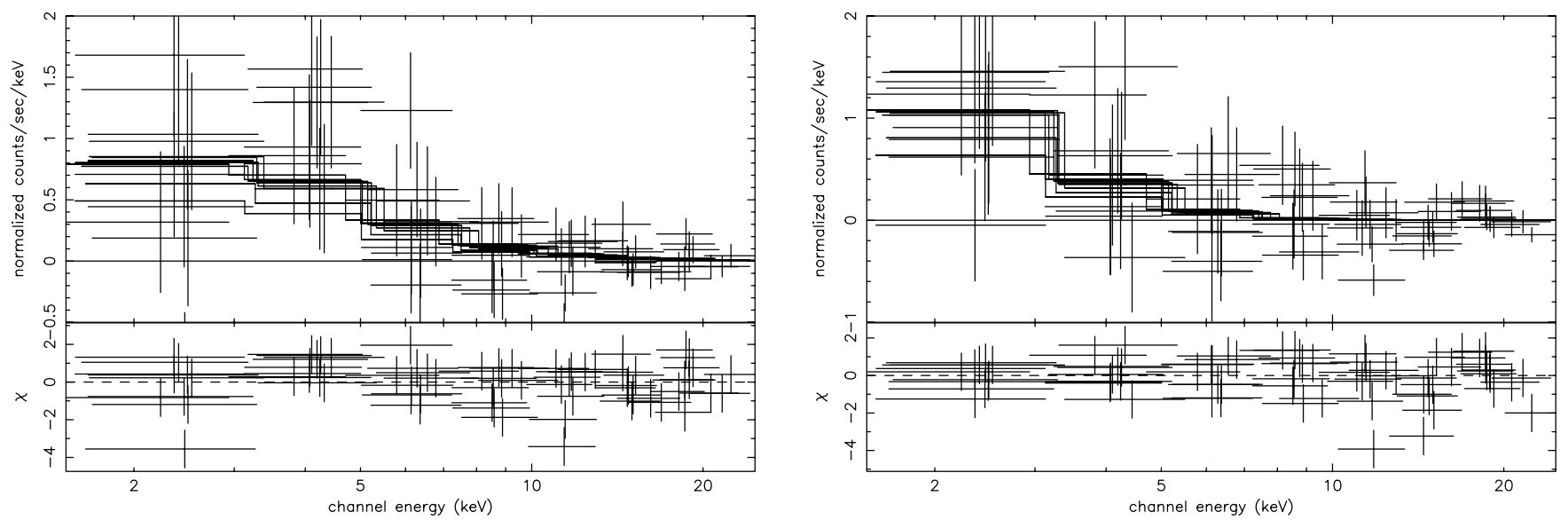

FIG. 3.-Comparison of the WXM spectra for the time intervals $0-8$ and 8-13 s. The observed (crosses) and predicted (histogram) count rates are shown in a different color for each of the nine WXM wires that we have included in the fits. The spectral model is a power law with fixed photoelectric absorption (see Table 2). [See the electronic edition of the Journal for a color version of this figure.]

law model, for the time intervals $0-8$ and $8-13$ s. Figure 4 shows the same comparison, except for the total duration of the burst $(0-13 \mathrm{~s})$. Table 3 gives the peak photon number and energy fluxes (in 1 s) and the fluence of XRF 020903, assuming the power-law model. ${ }^{20}$

Using the power-law model that best fits the burst-averaged WXM plus FREGATE spectral data, we find fluences $S_{\mathrm{X}}(2-$ $30 \mathrm{keV})=8.2_{-2.3}^{+2.5} \times 10^{-8} \mathrm{ergs} \mathrm{cm}^{-2}$ and $S_{\gamma}(30-400 \mathrm{keV})=$ $1.6_{-1.3}^{+4.2} \times 10^{-8} \mathrm{ergs} \mathrm{cm}^{-2}$, where the quoted errors give the $90 \%$ confidence intervals. Thus the ratio of fluences $\log \left[S_{\mathrm{X}}(7-\right.$ $\left.30 \mathrm{keV}) / S_{\gamma}(30-400 \mathrm{keV})\right]=0.7$, with a $90 \%$ lower limit of 0.3 , making this burst not only an XRF but also the most extreme example of an XRF observed so far by HETE-2.

A comparison of the power-law and Band-function fits to the first peak, which has a much higher $\mathrm{S} / \mathrm{N}$ than the second peak, provides modest evidence for an $E_{\text {peak }}^{\text {obs }}$ near $2 \mathrm{keV}$, the lower limit of the energy range of the WXM detectors. Specifically, we find that $\Delta \chi^{2}=4.34$ for one additional parameter, which means that the data requests the (more complicated) Band-function model at the $89 \%$ confidence level. However, the evidence is clearly not of high statistical significance, and in this fit we fixed $\alpha$ at -1 , its typical value for GRBs.

We therefore choose to place an upper limit on $E_{\text {peak }}^{\text {obs }}$. The appropriate model to use is the Band function, since (as we have already mentioned) the spectra of almost all GRBs and at least some XRFs are well described by this function. However, this presents a problem: the Band function has two distinct ways of representing a power-law spectrum in the detector energy range. First, it can do so by having $E_{\text {break }} \rightarrow 0$, so that only the high-energy, pure power-law part of the Band function is visible in the energy range of the detector. Second, it can do so by having $E_{\text {break }} \rightarrow \infty$ and $E_{0} \rightarrow \infty$, where $E_{0}$ is the "cutoff energy" of the cutoff power law that constitutes

\footnotetext{
${ }^{20}$ We compute the peak photon number flux in the WXM 2-5, 5-10, and $2-10 \mathrm{keV}$ energy bands, using the best-fit power-law model parameters for the average photon energy spectrum of the burst and the ratio 2.731 of the photon flux in the $1 \mathrm{~s}$ time interval containing the largest number of photons and the average photon flux in the $0-13 \mathrm{~s}$ time interval. We compute the peak photon energy flux in the WXM $2-5,5-10$, and $2-10 \mathrm{keV}$ energy bands in exactly the same way, except that we use the ratio 3.247 of the total photon energy flux (found by weighting each photon with its energy and summing the energies) found in the $1 \mathrm{~s}$ time interval containing the largest total photon energy and the average photon energy flux in the $0-13 \mathrm{~s}$ time interval.
}

the low-energy part of the Band function. In this limit, the limiting power law is actually the cutoff power law, but the cutoff energy is so large that the curvature of the model is imperceptible in the detector energy range.

We solve this problem by developing a new statistical method. This method uses a constrained Band function that is parameterized by two quantities, $E_{\text {peak }}^{\text {obs }}$ and $\beta$. The constrained Band function is perfectly able to make both pure power-law spectra and power law times exponential spectra of the required curvature in the detector energy range, but only the high-energy part of the Band function is allowed to produce a pure power-law spectrum. We describe this new method in detail in the Appendix. This method has general applicability to all instruments when the spectra of the bursts considered have $E_{\text {peak }}^{\text {obs }}$ near or below the low-energy threshold of the detector. It is necessary to demonstrate that the photon index $\beta<-2$ before applying this method.

In applying the constrained Band-function method to XRF 020903, we jointly fit the WXM and the FREGATE data. Figure 5 shows the posterior probability density distribution for $E_{\text {peak }}^{\text {obs }}$ that we find using this approach. From this posterior probability density distribution, we find a best-fit value

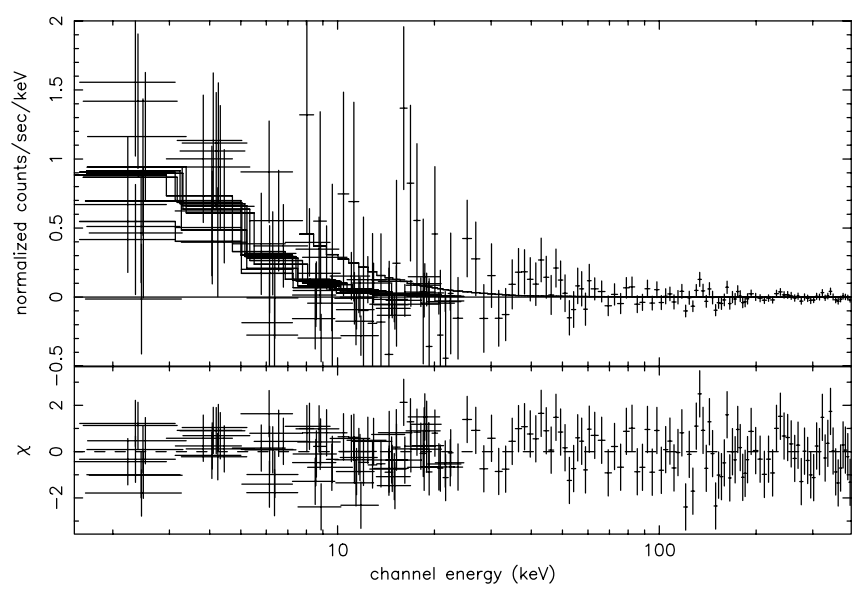

FIG. 4.-WXM and FREGATE spectra for the entire time interval $0-13 \mathrm{~s}$. The observed (crosses) and predicted (histogram) count rates are shown in a different color for each of the nine WXM wires that we have included in the fits. The spectral model is a power law with fixed photoelectric absorption (see Table 2). [See the electronic edition of the Journal for a color version of this figure.] 
TABLE 3

Peak Photon Number and Energy Fluxes (in 1 s) and Fluences in Various ENERGY BANDS FOR XRF 020903

\begin{tabular}{|c|c|c|c|}
\hline & $2-5 \mathrm{keV}$ & $5-10 \mathrm{keV}$ & $2-10 \mathrm{keV}$ \\
\hline Peak flux (photons $\mathrm{cm}^{-2} \mathrm{~s}^{-1}$ ).... & $1.9 \pm 0.7$ & $0.33_{-0.16}^{+0.19}$ & $2.2 \pm 0.8$ \\
\hline Peak flux $\left(10^{-9} \mathrm{ergs}^{-2} \mathrm{~s}^{-1}\right) \ldots \ldots$ & $10.4_{-3.7}^{+3.6}$ & $4.3_{-2.2}^{+2.3 .10}$ & $14.7 \pm 5.3$ \\
\hline Total fluence $\left(10^{-8} \mathrm{ergs} \mathrm{cm}^{-2}\right) \ldots \ldots$ & $4.2 \pm 0.9$ & $1.7_{-0.7}^{+0.8}$ & $5.9 \pm 1.4$ \\
\hline
\end{tabular}

Notes.-All of the quantities in this table are derived assuming a power-law model for the spectrum. The quoted errors correspond to the $90 \%$ confidence region.

$E_{\text {peak }}^{\text {obs }}=2.7 \mathrm{keV}$, that $1.1 \mathrm{keV}<E_{\text {peak }}^{\text {obs }}<3.6 \mathrm{keV}$ with $68 \%$ probability, and that $E_{\text {peak }}^{\text {obs }}<4.1$ and $5.0 \mathrm{keV}$ with $95 \%$ and $99.7 \%$ probabilities, respectively.

We conclude that the properties of XRF 020903 are very similar to those of long GRBs, with the exception that the observed peak energy $E_{\text {peak }}^{\text {obs }} \sim 3 \mathrm{keV}$ is $\sim 100$ times smaller. The extremely low value of $E_{\text {peak }}^{\text {obs }}$ seen in XRF 020903 is similar to the smallest value found among the nine XRFs whose spectra were determined by jointly fitting BeppoSAX WFC and BATSE data (Kippen et al. 2002).

\section{DISCUSSION}

\subsection{Source Properties}

We exclude the possibility that XRF 020903 is a type I X-ray burst (XRB) on the following grounds. First, its Galactic latitude is $b=-61.5$ (using the center of the combined WXM plus SXC error box), and there is no known persistent X-ray source or globular cluster in this error box. Since Type I X-ray burst sources lie in the Galactic plane or in globular clusters and have persistent X-ray emission, XRF 020903 is unlikely to be an X-ray burst on locational grounds alone. Second, the time history of XRF 020903 is not FRED-like (i.e., it does not exhibit a fast rise and an exponential decay), while those

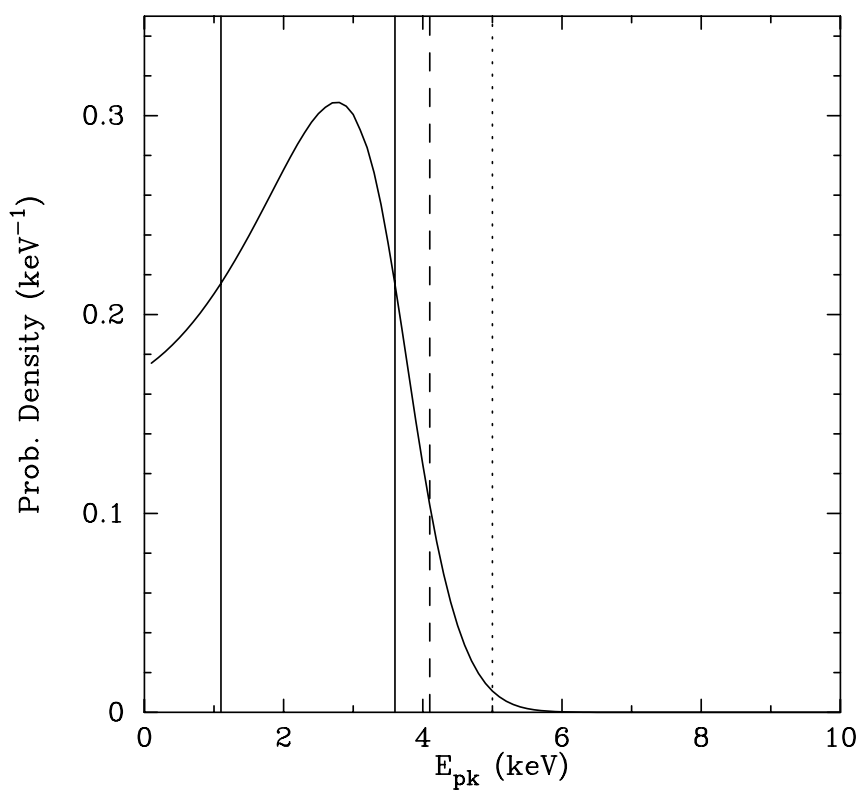

Fig. 5.-Posterior probability density distribution for $E_{\text {peak }}^{\text {obs }}$. The vertical solid lines define the $68 \%$ probability interval for $E_{\text {peak }}^{\text {obs }}$, while the dashed and dotted lines show the $95 \%$ and $99.7 \%$ probability upper limits on $E_{\text {peak }}^{\text {obs }}$. We find a best-fit value $E_{\text {peak }}^{\text {obs }}=2.7 \mathrm{keV}$, that $1.1 \mathrm{keV}<E_{\text {peak }}^{\text {obs }}<3.6 \mathrm{keV}$ with $68 \%$ probability, and that $E_{\text {peak }}^{\text {obs }}<4.1$ and $5.0 \mathrm{keV}$ with $95 \%$ and $99.7 \%$ probability. [See the electronic edition of the Journal for a color version of this figure.] of XRBs typically are. Third, although the blackbody model gives an acceptable fit to the spectra of the first and second peaks in the time history of XRF 020903, the derived blackbody temperatures are $\sim 1.0 \mathrm{keV}$. These temperatures are lower than those of almost all type I X-ray bursts (which typically have temperatures $T \approx 2 \mathrm{keV}$; see, e.g., Lewin, van Paradijs, \& Taam 1993). For these reasons, we conclude that XRF 020903 is an XRF and not an XRB.

The extremely low value of $E_{\text {peak }}^{\text {obs }}$ observed for XRF 020903 is remarkable. If the observed spectrum of XRF 020903 were the redshifted spectrum of a typical GRB, the implied redshift would be $z \sim 100$, using the best-fit value of $E_{\text {peak }}^{\text {obs }}=2.7 \mathrm{keV}$ observed for XRF 020903 and the mean value of $E_{\text {break }}^{\text {obs }}$ for the sample of 5500 spectra formed from the brightest 156 BATSE GRBs (Preece et al. 2000). A redshift of this magnitude would be hard to understand and is certainly not expected if long GRBs are associated with the collapse of massive stars (Lamb \& Reichart 2000). It is also wildly inconsistent with the measured redshift $z=0.25$ of the host galaxy (Soderberg et al. 2002) of the candidate optical (Soderberg et al. 2002) and radio (Berger et al. 2002) afterglows of XRF 020903. It is therefore difficult to attribute the low observed value of $E_{\text {peak }}^{\text {obs }}$ for XRF 020903 to cosmological redshift.

\subsection{Fluence and Peak Energy Correlations}

In Figure 6 we plot XRF 020903 in the $\left(S_{30-400}, S_{7-30}\right)$ plane, where $S_{7-30}$ and $S_{30-400}$ are the energy fluences of the bursts in the 7-30 and 30-400 keV energy bands. For the

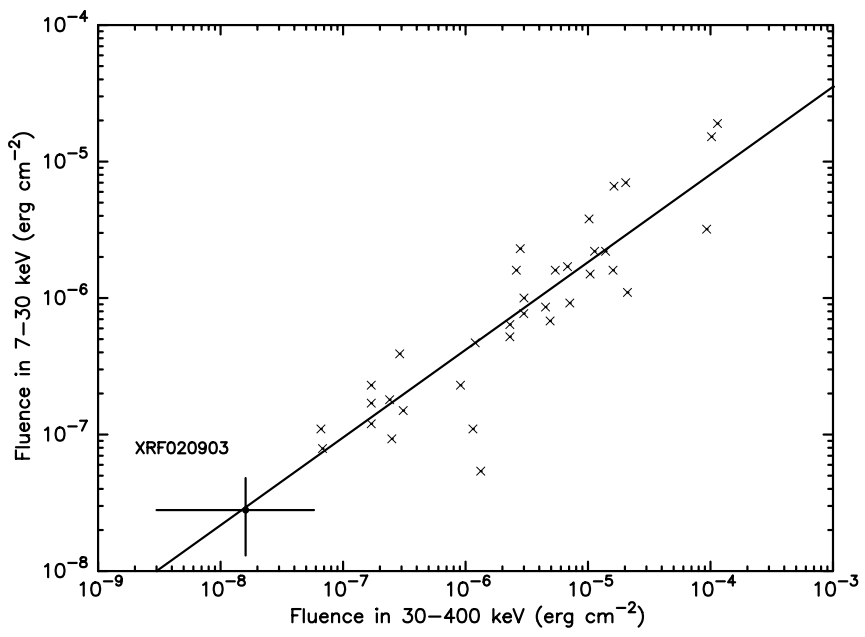

FIG. 6.- $\left(S_{7}-30, S_{30}-400\right)$-plane, showing the location of XRF 020903, using the total fluence in the 7-30 keV energy band and in the $30-400 \mathrm{keV}$ energy band (filled circle). The crosses are the locations of the 35 HETE/FREGATE GRBs studied by Barraud et al. (2003). The solid line is the relation, $S_{7-30}=$ $3 \times 10^{-3} \mathrm{~S}_{30-400}^{0.643}$, found by Barraud et al. (2003). The properties of XRF 020903 are consistent with this relation. 
value of $S_{7-30}$ and $S_{30-400}$, we use the best-fit power-law model for the average spectrum of WXM and FREGATE. Also plotted in this figure are the 35 GRBs whose spectra have been determined using HETE-2 FREGATE data (Barraud et al. 2003). Figure 6 shows that the properties of XRF 020903 are consistent with the relation between $S_{7-30}$ and $S_{30-400}$ found by Barraud et al. (2003).

In Figure 7 we plot XRF 020903 in the $\left(S_{2-400}, E_{\text {peak }}^{\text {obs }}\right)$-plane, where $E_{\text {peak }}^{\text {obs }}$ is the peak of the observed $\nu F_{\nu}$ spectrum. For $E_{\text {peak }}^{\text {obs }}$ we plot the $99.7 \%$ upper limit $(5.0 \mathrm{keV})$. The properties of XRF 020903 are consistent with an extension by two decades of the hardness-intensity correlation (Mallozzi et al. 1995; Lloyd-Ronning, Petrosian, \& Mallozzi 2000) between $S_{30-400}$ and $E_{\text {peak }}^{\text {obs }}$ found by Barraud et al. (2003).

Amati et al. (2002) demonstrated that there is a relation between $E_{\text {iso }}$ and the burst-averaged value of $E_{\text {peak }}$ (i.e., $E_{\text {peak }}$ for the time-averaged spectrum of the burst). Assuming that the candidate optical and radio afterglows of XRF 020903 are indeed the afterglows of XRF 020903, and therefore that the redshift of the underlying host galaxy is the redshift of the XRF, we can calculate the isotropic-equivalent radiated energy $E_{\text {iso }}$ and the upper limit on the burst-averaged peak energy $E_{\text {peak }}$ of the $\nu F_{\nu}$ spectrum in the source frame in the same way as did Amati et al. (2002). Figure 8 shows that the properties of XRF 020903 are consistent with an extension by a factor of $\sim 300$ in $E_{\text {iso }}$ of the relation found by Amati et al. (2002).

Figures 6-8 provide evidence that XRFs, "X-ray-rich GRBs," and GRBs form a continuum and are therefore the same phenomenon.

\subsection{Constraints on Theoretical Models of XRFs}

A variety of theoretical models have been proposed to explain XRFs (see, e.g., Zhang \& Mészáros 2003 for a comparative discussion of several of these models). In the off-axis GRB jet model (Yamazaki, Ioka, \& Nakamura 2002, 2003), XRFs are the result of viewing the jet of an ordinary GRB offaxis, so that relativistic beaming shifts the $\gamma$-rays into the

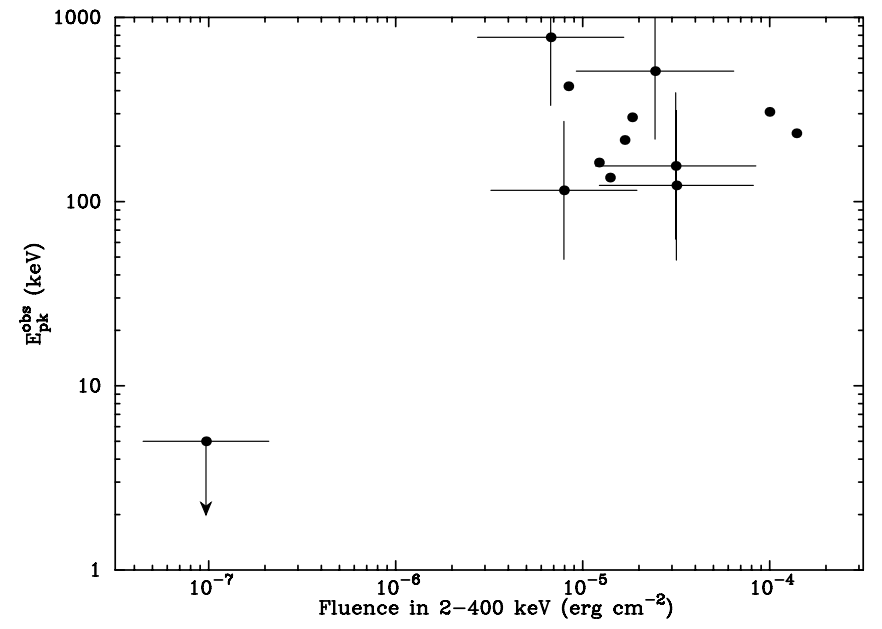

FIG. 7.- $\left(S_{2}-400, E_{\text {peak }}^{\text {obs }}\right)$-plane, showing the location of XRF 020903 . For $E_{\text {peak }}^{\text {obs }}$, we plot the $99.7 \%$ probability upper limit $(5.0 \mathrm{keV})$. We calculate the 2-400 keV fluence using the best power-law model fit jointly to the WXM and the FREGATE data. The crosses show the locations of 12 of the HETE-2 GRBs studied by Barraud et al. (2003) for which $E_{\text {peak }}^{\text {obs }}$ is relatively well determined. The properties of XRF 020903 are consistent with an extension by two decades of the hardness-intensity correlation found by Barraud et al. (2003). [See the electronic edition of the Journal for a color version of this figure.]

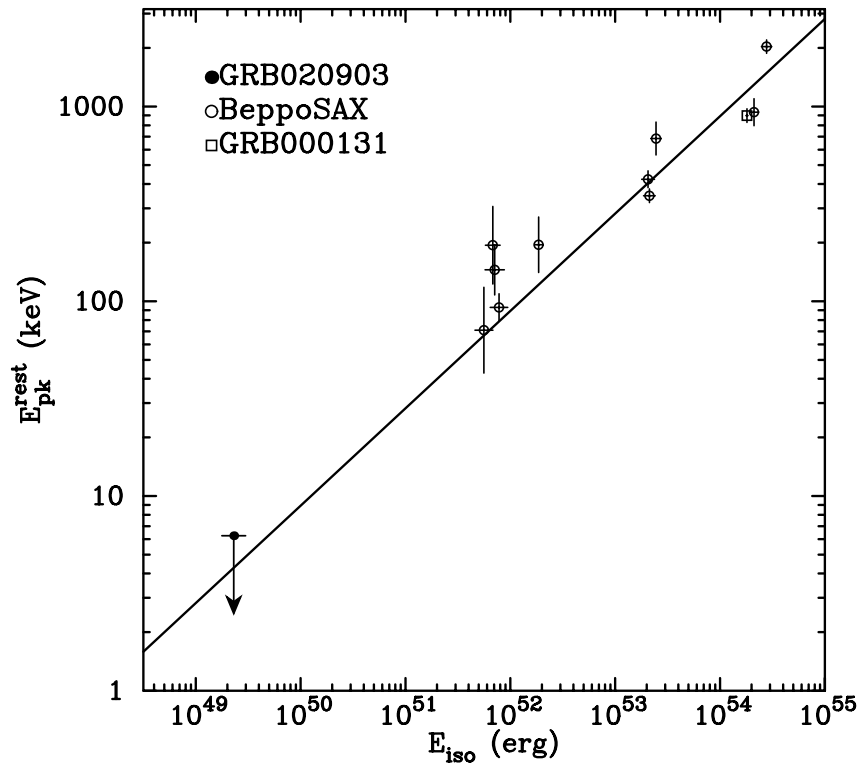

FIG. 8.- $\left(E_{\text {iso }}, E_{\text {peak }}\right)$-plane, where $E_{\text {iso }}$ is the isotropic-equivalent radiated energy between $1-10^{4} \mathrm{keV}$ and $E_{\text {peak }}$ is the peak of the $\nu F_{\nu}$ spectrum, both measured in the rest frame of the burst. The filled circle in the lower left-hand corner is the location of XRF 020903. The 10 open circles are the BeppoSAX GRBs reported by Amati et al. (2002). The solid line is given by the equation, $E_{\text {peak }}=89\left(E_{\text {iso }} / 10^{52} \mathrm{ergs}\right)^{0.5} \mathrm{keV}$. The properties of XRF 020903 are consistent with an extension of this relation by a factor $\sim 300$. [See the electronic edition of the Journal for a color version of this figure.]

X-ray range. In the clean fireball model, XRFs are due to the relativistic pair plasma in the GRB jet becoming optically thin much later than usual, at which time the relativistic bulk Lorentz factor $\Gamma$ has already decreased to a relatively low value (Mochkovitch et al. 2003). In the dirty fireball model, XRFs occur when there is significant baryon loading of the GRB jet, so that $\Gamma$ never reaches large values (Dermer, Chiang, \& Böttcher 1999; Huang, Dai, \& Lu 2002). In the universal jet model, XRFs are the result of viewing the GRB jet off-axis, where $\Gamma$ is lower because of the structure of the jet (Rossi, Lazzati, \& Rees 2002; Woosley, Zhang, \& Heger 2003; Zhang \& Mészáros 2002; Mészáros, Ramirez-Ruiz, Rees, \& Zhang 2002). In the uniform jet model, the different properties of XRFs, "X-ray-rich" GRBs, and GRBs are due primarily to different jet opening angles, with larger jet opening angles associated with lower values of $\Gamma$ (Lamb, Donaghy \& Graziani 2003).

Any such model of XRFs must reproduce the correlation found by Barraud et al. (2003) between $S_{7-30}$ and $S_{30-400}$ and the evidence we report in this paper for correlations between $S_{2-400}$ and $E_{\text {peak }}^{\text {obs }}$, and especially $E_{\text {iso }}$ and $E_{\text {peak }}$-the latter spanning nearly five decades in $E_{\text {iso }}$.

\section{CONCLUSIONS}

In this paper we have reported HETE-2 WXM/FREGATE observations of the X-ray flash, XRF 020903. This event was extremely soft: the spectrum had a best-fit peak energy $E_{\text {peak }}^{\text {obs }}=2.7 \mathrm{keV}$ and $E_{\text {peak }}^{\text {obs }}<5.0 \mathrm{keV}(99.7 \%$ probability upper limit), and no photons were detected above $\sim 10 \mathrm{keV}$. The burst is shorter at higher energies, which is typical of long GRBs. We considered the possibility that the burst lies at very high redshift and that the low value of $E_{\text {peak }}^{\text {obs }}$ is therefore due to the cosmological redshift: we showed that this is very unlikely. We find that the properties of XRF 020903 are consistent with the relation between $S_{7-30}$ and $S_{30-400}$ found by 
Barraud et al. (2003) for GRBs and X-ray-rich GRBs and with an extension by two decades of the hardness-intensity correlation (Mallozzi et al. 1995; Lloyd-Ronning et al. 2000) between $S_{30-400}$ and $E_{\text {peak }}^{\text {obs }}$ demonstrated by the same authors. Assuming that XRF 020903 lies at a redshift $z=0.25$, as implied by the host galaxy of the candidate optical afterglow of this burst, we find that the properties of XRF 020903 are consistent with an extension by a factor $\sim 300$ of the relation between $E_{\text {iso }}$ and $E_{\text {peak }}$ in the source frame of the burst found by Amati et al. (2002) for GRBs. When combined with earlier results, the results reported in this paper provide strong evidence that XRFs, X-ray-rich GRBs, and GRBs form a continuum and are a single phenomenon. The correlation found by Barraud et al. (2003) between $S_{7-30}$ and $S_{30-400}$ and the evidence we find in this paper for correlations between $S_{30-400}$ and $E_{\text {peak }}^{\text {obs }}$, and especially, $E_{\text {iso }}$ and $E_{\text {peak }}$, provide strong constraints on any model of XRFs and X-ray-rich GRBs.

We would like to thank the anonymous referee for comments and suggestions that materially improved the paper. The HETE-2 mission is supported in the US by NASA contract NASW-4690; in Japan in part by the Ministry of Education, Culture, Sports, Science, and Technology Grant-in-Aid 12440063; and in France by CNES contract 793-01-8479. $\mathrm{K}$. Hurley is grateful for Ulysses support under contract JPL 958059 and for HETE-2 support under contract MIT-SC-R293291. G. Pizzichini acknowledges support by the Italian Space Agency. One of the authors (T. S.) is partially supported by the Junior Research Associate (JRA) program at RIKEN.

\section{APPENDIX}

\section{THE “CONSTRAINED” BAND FUNCTION FOR SOFT GRBs}

In the spectral analysis of GRBs, one occasionally encounters events (such as XRF 020903) that are so soft that they present themselves as pure power laws with power-law index $\beta<-2$ in the energy range of the detector. The natural interpretation of such spectra is that the break energy $E_{\text {break }}$ separating the two functional parts of the Band function is near or below the lower boundary of the detector energy range.

This situation creates a problem for fits of the Band function, in that the Band function has two distinct ways of conforming to a power law in the detector energy range:

1. $E_{\text {break }} \rightarrow 0$, so that only the high-energy, pure power-law part of the Band function is visible in the energy range of the detector.

2. $E_{\text {break }} \rightarrow \infty, E_{0} \rightarrow \infty$, where $E_{0}$ is the "cutoff energy" of the cutoff power law that constitutes the low-energy part of the Band function. In this limit, the limiting power law is actually the cutoff power law, but the cutoff energy is so large that the curvature of the model is imperceptible in the detector energy range.

Therefore, despite the fact that the numerical value of the power-law index is such that we are certain that we should be dealing with the high-energy part of the Band function (i.e., the index is less than -2), the low-energy part of the function can "horn in" on the fit, altering the physical inferences drawn from the spectrum.

This situation is particularly a problem for the estimation of $E_{\text {peak }}$. Since we know that we are in case 1 , we also know that we ought to have at least a firm upper limit on $E_{\text {peak }}$, since $E_{\text {peak }}$ is always necessarily less than $E_{\text {break }}$, which is at the low end of the detector energy range. On the other hand, the case 2 limit implies $E_{\text {peak }} \rightarrow \infty$. Unfortunately, the data do not care which side of the Band function makes the power law, so no discrimination is possible between the two cases. Consequently, we cannot constrain $E_{\text {peak }}$ at all using a normal Band-function fit.

The approach we have chosen to deal with this situation in the case of XRF 020903 is to fit a constrained Band function to the data. That is, we consider a three-dimensional subspace of the full four-dimensional Band-function parameter space, choosing the subspace with a view to satisfying the following criteria:

1. It is perfectly possible to make both pure power laws and cutoff power laws of the any desired curvature in the detector energy range.

2. Only the high-energy part of the Band function is allowed to produce a pure power law.

We define the three-dimensional subspace in the following way: consider a Band function parameterized by low- and highenergy indices $\alpha$ and $\beta$, and by a cutoff energy $E_{0}$. The well-known relation between $E_{0}$ and $E_{\text {break }}$ is $E_{\text {break }}=(\alpha-\beta) E_{0}$. We impose the constraint condition on our family of fitting functions

$$
E_{\text {break }}=E_{\text {pivot }}\left(E_{0} / E_{\text {pivot }}\right)^{-1}
$$

where $E_{\text {pivot }}$ is some suitably chosen energy, in the general neighborhood where the GRB has appreciable emission. $E_{\text {break }}$ and $E_{0}$ are then inversely related, and are equal to each other when both are equal to $E_{\text {pivot }}$.

When $E_{0}<E_{\text {pivot }}$, then $E_{\text {break }}>E_{\text {pivot }}$, and the function is essentially a cutoff power law in the energy range of interest.

On the other hand, when $E_{0}>E_{\text {pivot }}$, then $E_{\text {break }}<E_{\text {pivot }}$, and as $E_{0} \rightarrow \infty, E_{\text {break }} \rightarrow 0$. In other words, when the low-energy part of the Band function is trying to imitate a power law, the break energy becomes small enough to force the low-energy part of the function below the energy range of interest, where it cannot be seen and therefore can do no harm. Any pure power-law work must thus be done by the high-energy part of the Band function.

The resulting spectral function has three parameters (including the scale), rather than four. The two input shape parameters can be chosen arbitrarily from the set $\left\{\alpha, \beta, E_{0}, E_{\text {break }}, E_{\text {peak }}\right\}$. The remaining parameters may then be determined by algebraic relationships. 
We have found it most convenient to adopt $E_{\text {peak }}$ and $\beta$ as our parameters. The choice of $E_{\text {peak }}$ is dictated by the necessity of estimating its value, or at least an upper bound on its value. The choice of $\beta$ is convenient because one may then impose the parameter bound $\beta<-2$, which guarantees that the formal expression for $E_{\text {peak }}$ may be meaningfully interpreted as the energy of the peak of the $\nu F_{\nu}$ distribution. This bound on $\beta$ is an important part of the specification of the fitting family of models. Were it not imposed, it would be possible for the formal expression for $E_{\text {peak }}$ to exceed $E_{\text {break }}$, so that at large values of $E_{\text {peak }}$ the fit could always produce a $\beta \gtrsim-2$ power law in the detector energy range. The result would be an extended tail of constant $\chi^{2}$ for arbitrarily large values of $E_{\text {peak }}$.

Figure 9 shows the constrained Band function, with $\beta=-2.5$ and $E_{\text {pivot }}=4 \mathrm{keV}$, for different values of $E_{\text {peak. }}$. This figure shows that $E_{\text {peak }}$ increases, $E_{\text {break }}$ also necessarily increases, so that $E_{0}$ is forced to smaller and smaller values by the constraint, which increases the curvature (and the value of $\alpha$ ).

Figure 10 shows the constrained Band function, with $E_{\text {peak }}=4 \mathrm{keV}$ and $E_{\mathrm{pivot}}=4 \mathrm{keV}$, for different values of $\beta$. The progression from some curvature at low energy $(\beta=-2.0)$ to almost none $(\beta=-4.0)$ is evident, as is the fact that as the curvature disappears, the resulting power law is produced by the high-energy part of the Band function.

Figures 9 and 10 show that the constrained Band function is perfectly able to make both pure power laws, and cutoff power laws with any desired curvature in the detector energy range. Figure 10 demonstrates that, in the constrained Band function, a power-law spectrum is always produced by the high-energy part of the Band function.

The choice of $E_{\text {pivot }}$ is dictated by the following considerations:

1. $E_{\text {pivot }}$ must be low enough to prevent the low-energy part of the Band function from making a power law in the energy range of interest. If $E_{\text {pivot }}$ were $1 \mathrm{GeV}$ (say), then the Band function would have no difficulty making $E_{0}$ large and $\alpha \lesssim-2$, which is what we are trying to prevent by introducing the constraint. So $E_{\text {pivot }}$ should be "as low as possible."

2. $E_{\text {pivot }}$ must not be so low that we cannot adequately fit any curvature that may exist in the spectrum. If $E_{\text {pivot }}$ were $1 \mathrm{eV}$ (say), then whenever $E_{\text {break }}$ was in or above the energy range where the spectrum is appreciable, $E_{0}$ would be so tiny that the curvature of the model would be huge, much too large to fit the data well.

One way of choosing $E_{\text {pivot }}$ is to calculate its value using the best-fit parameters from a fit of a free Band function, using $E_{\text {pivot }}=\left(E_{0} E_{\text {break }}\right)^{1 / 2}$. This choice, which effectively chooses the unique constrained subspace of the full parameter space that contains the best-fit free Band function, allows the constrained family of functions to optimally fit whatever curvature the data may seem to hint is required.

We must require that the inferences that we draw from the spectral fit should be robust, in the sense that they should not depend strongly on the specific choice of $E_{\text {pivot }}$. So the proper use of this constrained Band model involves not only choosing a representative value of $E_{\text {pivot }}$, but also varying $E_{\text {pivot }}$ in some reasonable range, to make sure that the conclusions about parameter estimates and bounds are unaffected by the choice of $E_{\text {pivot}}$.

Figure 11 shows the constrained Band function, with $E_{\text {peak }}=4 \mathrm{keV}$ and $\beta=2.5$, for different values of $E_{\text {pivot. }}$ Once again, as the low-energy curvature disappears, the resulting power law is produced by the high-energy part of the Band function. Figure 11 also shows that the shape of the spectrum in the detector energy range is insensitive to the specific choice of $E_{\text {pivot, }}$, within a reasonable range. Thus the conclusions about parameter estimates and bounds are unaffected by the choice of $E_{\text {pivot }}$.

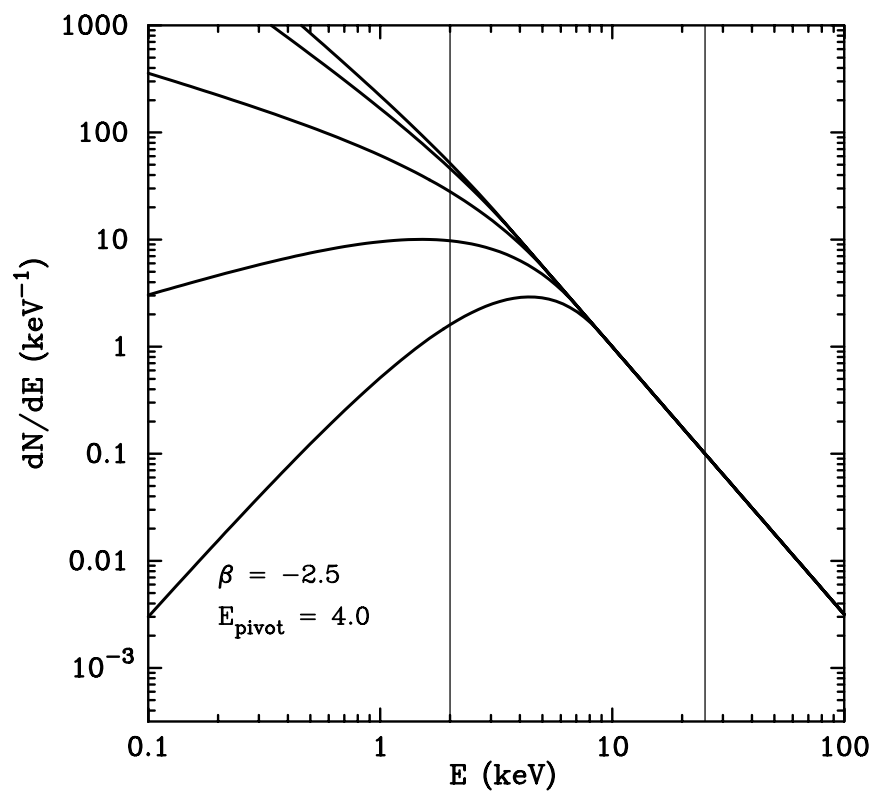

FIG. 9.- Constrained Band functions, with $\beta=-2.5$ and $E_{\text {pivot }}=4 \mathrm{keV}$, for different values of $E_{\text {peak. }}$ All functions have been normalized to $1 \mathrm{keV}-1$ at $10 \mathrm{keV}$. The two vertical lines at 2 and $25 \mathrm{keV}$ show the WXM bandpass. The spectra shown are (decreasing monotonically from the top at low energy), $E_{\text {peak }}=1,2,4,6$, and $8 \mathrm{keV}$, respectively. As $E_{\text {peak }}$ increases, $E_{\text {break }}$ also necessarily increases, so that $E_{0}$ is forced to smaller and smaller values by the constraint, increasing the curvature and the value of $\alpha$. 


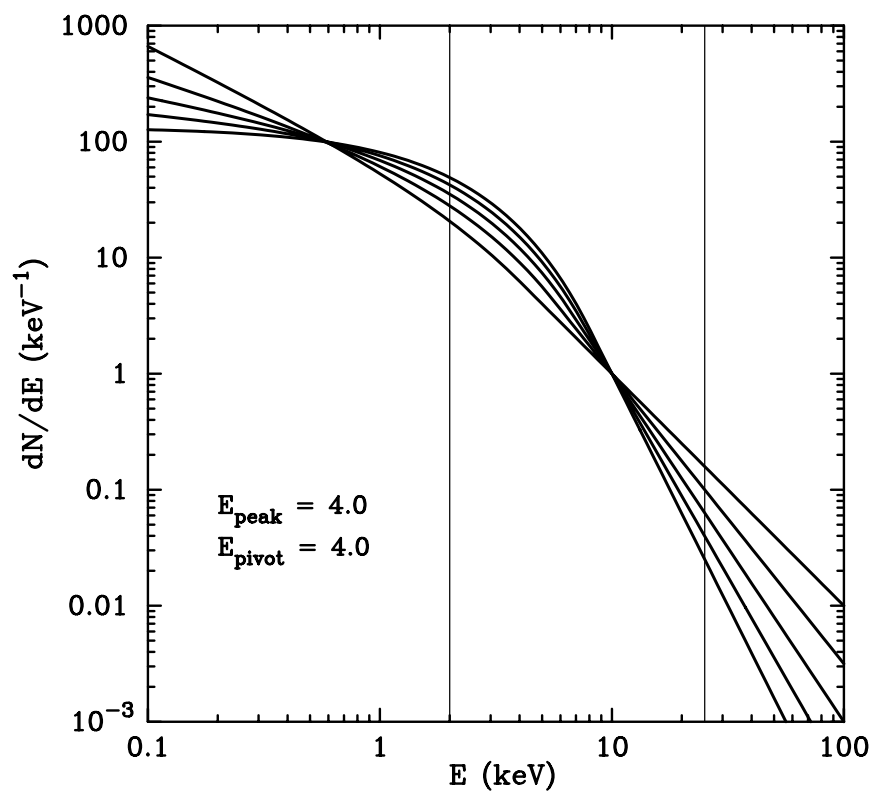

Fig. 10.-Constrained Band functions, with $E_{\text {peak }}=4 \mathrm{keV}$ and $E_{\text {pivot }}=4 \mathrm{keV}$, for different values of $\beta$. All functions have been normalized to $1 \mathrm{keV}^{-1}$ at $10 \mathrm{keV}$. The two vertical lines at 2 and $25 \mathrm{keV}$ show the WXM bandpass. The spectra shown are for $\beta=-2.0,-2.5,-3.0,-3.5$, and -4.0 , which can be distinguished by the increasing steepness of their slopes at high energy. The progression from some curvature at low energy $(\beta=-4.0)$ to almost none $(\beta=-2.0)$ is evident, as is the fact that as the curvature disappears, the resulting power-law is produced by the high-energy part of the Band function.

Figure 12 shows the constrained Band functions with parameters that best fit the $13 \mathrm{~s}$ spectrum of XRF 020903, for different fixed values of $E_{\text {pivot }}$. This figure illustrates the fact that the shape of the best-fit model is essentially unchanged in the energy range of the WXM for choices of $E_{\text {pivot }}$ within a reasonable range.

Finally, we give the algebraic relationships necessary to recover the remaining Band function parameters assuming that $E_{\text {peak }}$ and $\beta$ are given. Let $x \equiv E_{\text {peak }} / E_{\text {pivot. }}$ Then,

$$
\begin{aligned}
\alpha & =-2+\frac{1}{2} x^{2}+\sqrt{\frac{1}{4} x^{4}-x^{2}(\beta+2)}, \\
E_{0} & =(2+\alpha) E_{\text {peak }} .
\end{aligned}
$$

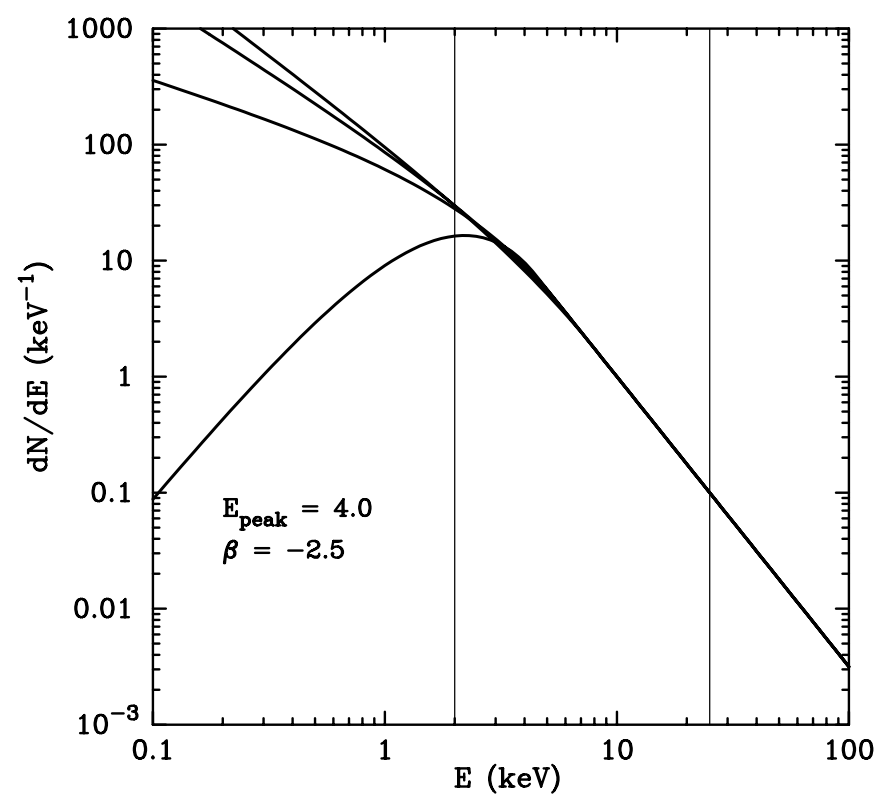

Fig. 11. Constrained Band functions, with $E_{\text {peak }}=4 \mathrm{keV}$ and $\beta=-2.5$, for different values of $E_{\text {pivot. }}$ All functions have been normalized to $1 \mathrm{keV}-1$ at $10 \mathrm{keV}$. The two vertical lines at 2 and $25 \mathrm{keV}$ show the WXM bandpass. The spectra shown are (increasing monotonically at low energy) for $E_{\text {pivot }}=2,4,6$, and $8 \mathrm{keV}$, respectively. Once again, as the low-energy curvature disappears, the resulting power-law is produced by the high-energy part of the Band function. Note also that the shape of the constrained Band function is insensitive to the specific choice of $E_{\text {pivot }}$ within a reasonable range. 


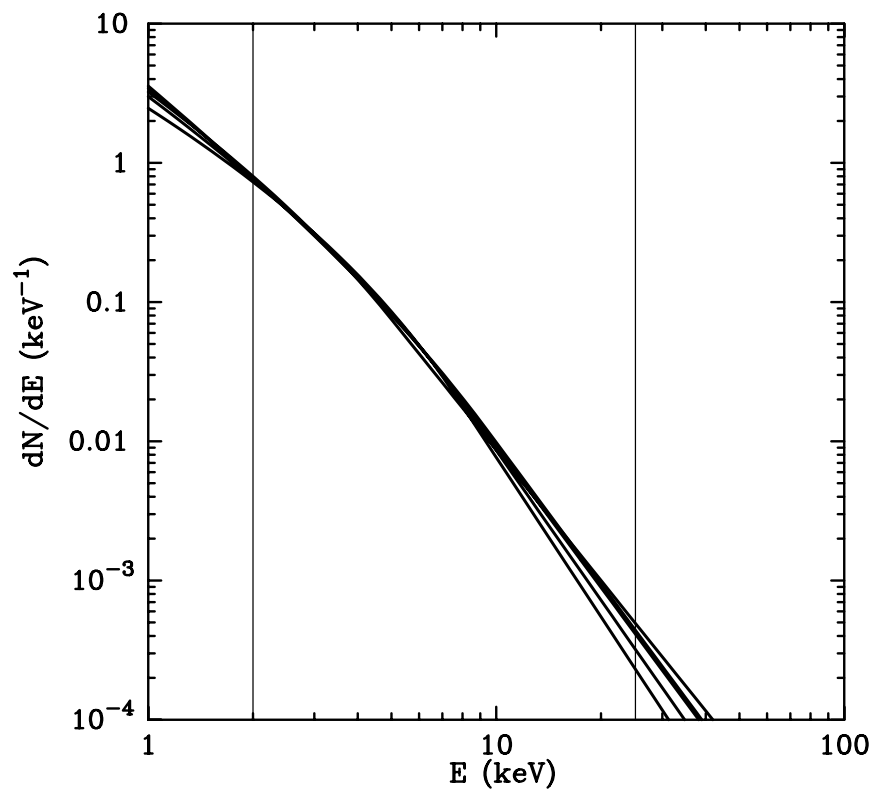

FIG. 12.-Constrained Band functions with parameters that best fit the $13 \mathrm{~s}$ spectrum of XRF 020903, for different fixed values of $E_{\text {pivot. }}$ The two vertical lines at 2 and at $25 \mathrm{keV}$ show the WXM bandpass. All functions have been normalized so that the integral from 2 to $25 \mathrm{keV}$ is one photon. The five spectra shown in the plot corresponding to $E_{\text {pivot }}=4,5,6,7$, and $8 \mathrm{keV}$ (the 7 and $8 \mathrm{keV}$ ones largely overlap each other). This figure illustrates a robust aspect of the constraint procedure: the best-fit model is essentially unchanged in the WXM spectral band despite a factor of 2 change in the value of $E_{\text {pivot }}$.

In equation (A2), we have resolved the ambiguity in the choice of root of a quadratic equation by requiring that when $\beta+2<0$, then $\alpha+2>0$, so that $E_{\text {peak }}$ is in fact the peak energy of the $\nu F_{\nu}$ distribution.

\section{REFERENCES}

Amati, L., et al. 2002, A\&A, 390, 81

Atteia, J.-L., et al. 2003, in Gamma-Ray Bursts and Afterglow Astronomy, ed. G. R. Ricker \& R. Vanderspek (Melville: AIP), 17

Band, D. L., et al. 1993, ApJ, 413, 281

Barraud, C., et al. 2003, A\&A, 400, 1021

Berger, E., Kulkarni, S. R., Frail, D. A., Soderberg, Price, P. A., Fox, D. W., Harrison, F. A., \& Yost, S. 2002, GCN Circ. 1555

Chornock, R., \& Filippenko, A. V. 2002, GCN Circ. 1609

Covino, S., et al, 2002, GCN Circ. 1563

Dermer, C. D., Chiang, J., \& Böttcher, M. 1999, ApJ, 513, 656

Dickey, J. M., \& Lockman, F. J. 1990, ARA\&A, 28, 215

Fenimore, E. E., in 't Zand, J. J. M., Norris, J. P., Bonnell, J. T., \& Nemiroff, R. J. 1995, ApJ, 448, L101

Graziani, C., et al. 2003, in Gamma-Ray Bursts and Astronomy, ed. G. R. Ricker \& R. Vanderspek (Melville: AIP), 114

Heise, J., in 't Zand, J., Kippen, R. M., \& Woods, P. M. 2000, in Proc. Second Rome Workshop: Gamma-Ray Bursts in the Afterglow Era, ed. E. Costa, F. Frontera, \& J. Hjorth (Berlin: Springer), 16

Huang, Y. F., Dai, Z. G., \& Lu, T. 2002, MNRAS, 332, 735

Kawai, N., et al. 2003, in Gamma-Ray Bursts and Afterglow Astronomy, ed. G. R. Ricker \& R. Vanderspek (Melville: AIP), 25

Kippen, R. M., Woods, P. M., Heise, J., in 't Zand, J., Briggs, M. S., \& Preece, R. D. 2002, in Gamma-Ray Bursts and Afterglow Astronomy, ed. G. R. Ricker \& R. Vanderspek (Melville: AIP), 244

Lamb, D. Q., Donaghy, T. Q., \& Graziani, C. 2003a, ApJ, submitted (astro-ph/020615)

Lamb, D. Q., \& Reichart, D. E. 2000, ApJ, 536, 1

Levan, A., Fruchter, A., Strolger, L., Burud, I., \& Rhoads, J. 2002, GCN Circ. 1761

Lewin, W. H. G., van Paradijs, J.\& Taam, R. E. 1993, Space Sci. Rev., 62, 223

Lloyd-Ronning, N., Petrosian, V., \& Mallozzi, R. S. 2000, ApJ, 534, 227
Mallozzi, R. S., Paciesas, W. S., Pendleton, G. N., Briggs, M. S., Preece, R. D., Meegan, C. A., \& Fishman, G. J. 1995, ApJ, 454, 597

Mészáros, P., Ramirez-Ruiz, E., Rees, M. J., \& Zhang, B. 2002, ApJ, 578, 812

Mochkovitch, R., Daigne, F., Barraud, C., \& Atteia, J. L. 2003, preprint (astro-ph/0303289)

Monnelly, G., et al. 2003, in Gamma-Ray Bursts and Afterglow Astronomy, ed.

G. R. Ricker \& R. Vanderspek (Melville: AIP), 25

Paciesas, W. S., et al. 1999, ApJS, 122, 465

Preece, R. D., Briggs, M. S., Mallozzi, R. S., Pendleton, G. N., \& Paciesas, W. S. 2000, ApJS, 126, 19

Ricker, G. R., et al. 2002, GCN Circ. 1530 2003, in Gamma-Ray Bursts and Astronomy, ed. G. R. Ricker \& R. Vanderspek (Melville: AIP), 3

Rossi, E., Lazzati, D., \& Rees, M. J. 2002, MNRAS, 332, 945

Shirasaki, Y., et al. 2000, Proc. SPIE, 4012, 166 2002, Proc. SPIE, 4851, 1310

2003, in Gamma-Ray Bursts and Astronomy, ed. G. R. Ricker \& R. Vanderspek (Melville: AIP), 117

Soderberg, A. M., et al. 2002, GCN Circ. 1554

Strohmayer, T. E., Fenimore, E. E., Murakami, T., \& Yoshida, A. 1998, ApJ, 500,873

Villasenor, J. N., et al. 2003, in Gamma-Ray Bursts and Astronomy, ed. G. R. Ricker \& R. Vanderspek (Melville: AIP), 33

Woosley, S. E., Zhang, W., \& Heger, A. 2003, in AIP Conf Proc. 662, Gamma-Ray Burst and Afterglow Astronomy 2001, ed. G. R. Ricker \& R. K. Vanderspek (Melville: AIP) 185

Yamazaki, R., Ioka K., \& Nakamura T. 2002, ApJ, 571, L31 . 2003, ApJ, 593, 941

Zhang, B., \& Mészáros, P. 2002, ApJ, 571, 876

2003, ApJ, 581, 1236 